\title{
Elemental Composition of Analogs to Mars Return Samples Studied with X-Ray Fluorescence Imaging at NSLS-II.
}

\author{
$\underline{\text { Juergen Thieme }}^{1,}{ }^{*}$, Joel Hurowitz ${ }^{2}$, Martin Schoonen ${ }^{1,2}$, K. A. Farley ${ }^{3}$, S. Sherman ${ }^{3}$ and John Hill ${ }^{1}$. \\ 1. National Synchrotron Light Source II, Brookhaven National Laboratory, Upton, NY 11973, USA \\ 2. Department of Geosciences, Stony Brook University, Stony Brook, NY 11794, USA \\ 3. California Institute of Technology - Jet Propulsion Laboratory, Pasadena, CA 91109, USA \\ * Corresponding author, jthieme@bnl.gov
}

In 2020, NASA will launch out into the first step of what may be an ambitious, multi-mission campaign with the goal to bring back for analysis in laboratories here to Earth a collection of rock, regolith, and atmosphere samples from Mars. It is the main objective of this endeavor to determine whether or not Mars was ever host to ancient microbial life [1]. Finding evidence for extinct (or extant) life on Mars has the potential to fundamentally alter our view of the origin and evolution of life. It would provide proof for Earth being just one amongst others regarding the question of biological activity in the Solar System. Furthermore, returned Martian samples would provide a rich repository of information on the history of planetary accretion and differentiation, geochronology, atmospheric evolution, and the paleoenvironmental history of Mars, adding tremendous additional value to such a mission beyond the issue of extraterrestrial life [1]. The potential knowledge to be obtained by such a collection of samples will equal or exceed that of the Apollo lunar sample archive, which even today yields new insights on the history of the Solar System, over 40 years after they were returned to Earth (e.g., [2]).

Strictest Planetary Protection requirements will be stipulated to any laboratory working with Martian samples. These have been established to protect the Earth from potentially dangerous extant life associated with the returned Martian sample cache, as well as to prevent "false positives" for Martian life arising from terrestrial biological contamination [3]. Considering the timeline for creating procedures to work with Martian samples [4] and expecting samples returned from Mars in the early 2030 's, it is now time to provide proof-of-concept measurement data to establish the feasibility of sample handling and safe sample triage. The high X-ray brightness of the National Synchrotron Light Source-II (NSLS-II) at the Brookhaven National Laboratory provides a unique and critical capability to perform assessments of the elemental composition and the chemical state of Mars Return Samples using synchrotron radiation X-ray fluorescence imaging and X-ray absorption spectroscopy.

Six samples were collected using the coring drill prototype for the Mars 2020 Sample Caching System. These analog samples are Uniform Saddleback Basalt (USB), Bishop Tuff Intermediate (BTI), China Ranch Gypsum (CRG), Kramer Massive Mudstone (KMM), Napa Basaltic Sandstone (NBS), and Old Dutch Pumice (ODP). They were examined with the Sub-micron Resolution X-ray Spectroscopy beamline (SRX), creating X-ray fluorescence (XRF) maps, which are collected by a 3-element energy dispersive detector. XRF mapping and XANES imaging experiments to investigate the elemental composition and the chemical state of a sample with sub- $\mu \mathrm{m}$ spatial resolution are the core capabilities of the SRX beamline [5]. Within the X-ray energy range of about 5-20 keV, accessible to SRX, a wide range of elements show X-ray absorption edges, yielding detailed chemical information. XRF spectra were analyzed using the PyXRF software to determine elemental distribution and concentrations (Fig.1). Maps were created showing the heterogeneity within these analogs (Fig. 2). To investigate a Martian sample as a whole, the penetration ability of these X-ray energies is not enough. However, a shell or 
layer of the sample can be investigated. This will be possible for Martian return samples, when they are transferred into X-ray transparent sample containers. The experimental procedure is to transfer the samples into such a sleeve from the primary Ti-alloy tube under controlled conditions to avoid exposure to the terrestrial atmosphere. Materials such as $\mathrm{BeO}$ ceramic or polycarbonate are X-ray transparent in an X-ray energy range used for XRF measurements, allowing for an investigation by XRF techniques, well-established at NSLS-II. This has been shown in a proof-of-principle experiment, Fig. 3 shows the XANES result. XRF and XANES results and a detailed discussion will be presented.

Future work will include the collection of more chemical information for all samples, e.g. additional XRF maps to improve statistics and additional XANES spectra from elements present in the samples. The chemical coordination of a probed element can be determined using extended X-ray absorption fine structure (EXAFS) spectroscopy, which is another SRX capability to be applied to these samples. Additional proof-of-concept measurements will be conducted to determine the feasibility of phase contrast and to obtain XRF 3D information by confocal imaging. In addition, the effect of X-radiation on organic matter and cellular material at the intensities and exposures used in these techniques will be evaluated.

References:

[1] McLennan, S.M. et al., Final report of the MSR E2E-iSAG, MEPAG (2011) p. 101.

[2] McCubbin, F.M., et al., PNAS (2010) 107, 11223-11228.

[3] COSPAR (2002) The Quarantine and Certification of Martian Samples,

The National Academies Press.

[4] Smith, C., et al. (2016) iMARS Phase II: Findings and Recommendations, MEPAG Annual Meeting, Silver Spring, MD.

[5] Chen-Wiegart, Y.K., et al., Early science commissioning results of the sub-micron resolution X-ray spectroscopy beamline (SRX) in the field of materials science and engineering. (2016) http://doi.org/10.1063/1.4961138.

[6] Acknowledgements: This work was funded by an SBU-BNL seed grant to J. Hurowitz and J.

Thieme and DOE, Office of Science, Basic Energy Science under contract DE-SC0012704.

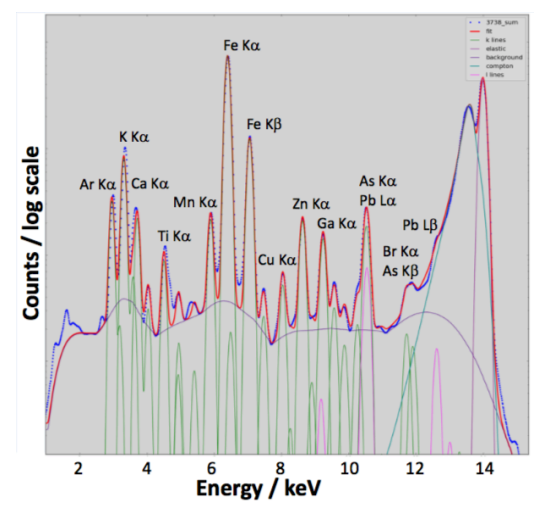

Figure. 1. X-ray fluorescence spectrum of BTI. Note that all elements are visible within just one scan, using here an inciden X-ray energy of $14 \mathrm{keV}$. Main fluorescence peaks are labeled.

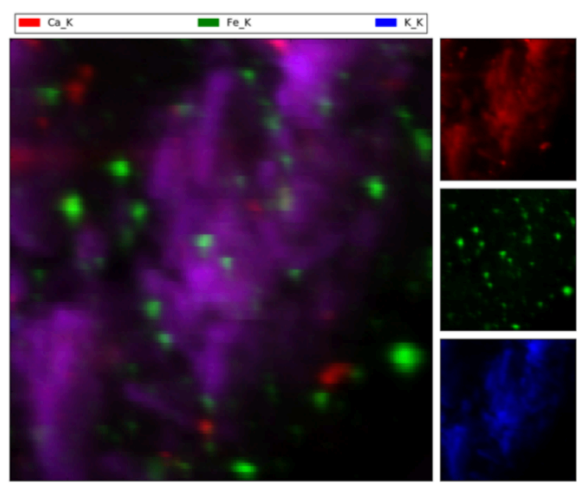

Figure. 2. RGB representation of $\mathrm{Ca}, \mathrm{Fe}$, and $\mathrm{K}$ in the BTI sample. Note the overlay of $\mathrm{Ca}$ and $\mathrm{K}$ in large parts. Size: $150 \times 150 \mu \mathrm{m}^{2}$, dwell time $0.3 \mathrm{~s}$, focal spot $=$ step size: $1 \mu \mathrm{m}^{2}$.

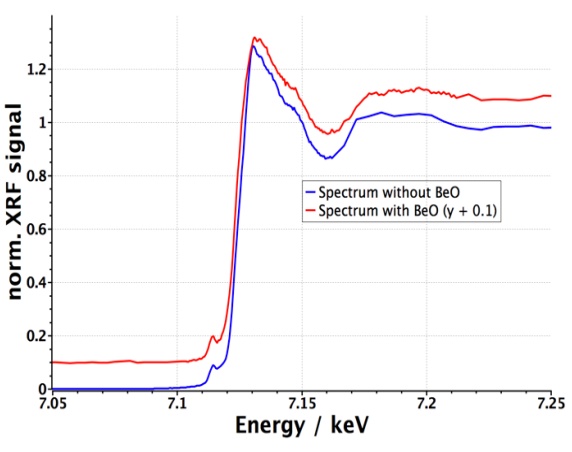

Figure. 3. XANES spectra of an iron-rich spot of a sample with and without $\mathrm{BeO}$ ceramic cover of $500 \mu \mathrm{m}$ thickness. The spectrum with $\mathrm{BeO}$ has been shifted in $\mathrm{y}$ for better comparison. 